\section{A Word from USDA}

In response to the questions posed in this scenario, the United States Department of Agriculture, Animal and Plant Health Inspection Service, Animal Care (USDA-APHIS-AC) offers the following clarification and guidance:

While we appreciate the investigators' decision to pursue a reduction in the number of animals likely to experience pain or distress, the process by which they went about it does not appear to be in compliance with the Animal Welfare regulations. Any significant change to an animal use proposal requires IACUC approval prior to the change being implemented. A new procedure, a change in the procedure being used, or adding a procedure may be considered 'significant'1. For example, the anesthetic regime (i.e., the refinement consideration) approved by the IACUC for each individual procedure may not have been adequate for the combined surgeries; therefore, the potential for the animal to experience pain or distress would be unacceptable. Both of these investigators' proposals must be amended, or consolidated, and approved by the IACUC before any further combined studies may be initiated.

In addition, we would suggest the facility review its training and instruction program to ensure all of the investigators understand the need for IACUC review, and procedures to obtain IACUC approval, in these situations.

\section{Reference}

1. USDA APHIS. Procedure for Protocol Review. Animal Care Resource Guide. (April 2001). http://www.aphis.usda.gov/ac/researchmanual/18-4PROC.PDF

Chester A. Gipson, DVM

Deputy Administrator

USDA-APHIS-Animal Care edge of that framework, and some of us might even bump against it and bounce off again. It is not acceptable to leave this framework, because it is one of the pillars upon which our society rests. And this is the case with our friends.

In Europe, the situation is far more obvious and therefore clearer to understand. Usually we do not have IACUCs. A governmental authority must give permission for every experimental work involving animals. Institutions have Animal Welfare Officers who act as liaisons between the scientists' institutions and the local authorities. The communication between the two parties goes through the Animal Welfare Officer's desk. The study Directors are responsible for their piece of work and are under supervision of the Animal Welfare Officer. The application gives a detailed description of the protocol. An Ethics Board composed of people either interested or educated in life sciences reviews the application. The Board functions in an advisory capacity to the Official Veterinarian, who in the end decides on the application. After the permission has finally been granted, the framework is set and clear to everybody involved.

Therefore, it is totally unacceptable in our part of the world to alter the protocol without giving notice to the authorizing agency and awaiting approval. If you break this rule, you'll have a federal case. It is not in the Animal Welfare Officer's authority to give an off-the-record 'heads up'. Our working field is too sensitive an area, and the framework in which we are working not only reduces our mobility and flexibility but also protects us from external disturbances. It is for ourselves as well as our employer of vital interest that we stay out of the focus of animal activists, and it is far easier for everybody to accept what we do if we adhere to the basic rules.

Josten is Head of Laboratory Animal Services, RCC Ltd, Fuellinsdorf, Switzerland. 